\title{
Binding of Sudan II and IV to lecithin liposomes and E. coli membranes: insights into the toxicity of hydrophobic azo dyes $\mathrm{Lu} \mathrm{Li}^{1}$, Hong-Wen Gao*1,2, Jiao-Rong Ren², Ling Chen¹, Yu-Cheng Li³ ${ }^{3}$, Jian- Fu Zhao' ${ }^{1}$ He-Ping Zhao ${ }^{1}$ and Yuan Yuan ${ }^{1,2}$
}

Address: ${ }^{1}$ State Key Laboratory of Pollution Control and Resource Reuse, College of Environmental Science and Engineering, Tongji University, Shanghai 200092, P. R. China, ${ }^{2}$ Key Laboratory of Yangtze Water Environment of Ministry of Education, College of Environmental Science and Engineering, Tongji University, Shanghai 200092, P. R. China and ${ }^{3}$ School of Life Science, Anhui University, Hefei 230039, P. R. China

Email: Lu Li - yaya1120@163.com; Hong-Wen Gao* - hwgao@mail.tongji.edu.cn; Jiao-Rong Ren - renjiaorong@163.com; Ling Chen - chenling@mail.tongji.edu.cn; Yu-Cheng Li - liyc1988@yahoo.com.cn; Jian-Fu Zhao - zhaojianfu@mail.tongji.edu.cn; HePing Zhao - hopechoil@hotmail.com; Yuan Yuan - enviklab@mail.tongji.edu.cn

* Corresponding author

Published: 27 March 2007

BMC Structural Biology 2007, 7:16 doi:10.1/86/1472-6807-7-16
Received: 20 December 2006

Accepted: 27 March 2007

This article is available from: http://www.biomedcentral.com/1472-6807/7/16

(C) $2007 \mathrm{Li}$ et al; licensee BioMed Central Ltd.

This is an Open Access article distributed under the terms of the Creative Commons Attribution License (http://creativecommons.org/licenses/by/2.0), which permits unrestricted use, distribution, and reproduction in any medium, provided the original work is properly cited.

\begin{abstract}
Background: Sudan red compounds are hydrophobic azo dyes, still used as food additives in some countries. However, they have been shown to be unsafe, causing tumors in the liver and urinary bladder in rats. They have been classified as category 3 human carcinogens by the International Agency for Research on Cancer. A number of hypotheses that could explain the mechanism of carcinogenesis have been proposed for dyes similar to the Sudan red compounds. Traditionally, investigations of the membrane toxicity of organic substances have focused on hydrocarbons, e.g. polycyclic aromatic hydrocarbons (PAHs), and DDT. In contrast to hydrocarbons, Sudan red compounds contain azo and hydroxy groups, which can form hydrogen bonds with the polar head groups of membrane phospholipids. Thus, entry may be impeded. They could have different toxicities from other lipophilic hydrocarbons. The available data show that because these compounds are lipophilic, interactions with hydrophobic parts of the cell are important for their toxicity. Lipophilic compounds accumulate in the membrane, causing expansion of the membrane surface area, inhibition of primary ion pumps and increased proton permeability.
\end{abstract}

Results: This work investigated the interactions of the amphiphilic compounds Sudan II and IV with lecithin liposomes and live Escherichia coli (E. coli). Sudan II and IV binding to lecithin liposomes and live E. coli corresponds to the Langmuir adsorption isotherm. In the Sudan red compounds - lecithin liposome solutions, the binding ratio of Sudan II to lecithin is I/3I and that of Sudan IV to I/3 I4. The binding constant of the Sudan II-lecithin complex is $1.75 \times 10^{4}$ and that of the Sudan IV-lecithin complex $2.92 \times 10^{5}$. Besides, the influences of $\mathrm{pH}$, electrolyte and temperature were investigated and analyzed quantitatively. In the Sudan red compounds - E.coli mixture, the binding ratios of Sudan II and Sudan IV to E.coli membrane phospholipid are I/29 and I/I I 4 . The binding constants of the Sudan II - and Sudan IV- E.coli membrane phospholipid complexes are $1.86 \times 10^{4}$ and $6.02 \times 10^{4}$. Over $60 \%$ of Sudan II and $75 \%$ of Sudan IV penetrated into E.coli, in which $90 \%$ of them remained in the E.coli membrane.

Conclusion: Experiments of Sudan II and IV binding to lecithin liposomes and live E. coli indicates that amphiphilic compounds may besequestered in thelecithin liposomes and membrane phospholipid bilayer according to the Langmuir adsorption law. Penetration into the cytosol was impeded and inhibited for Sudan red compounds. It is possible for such compounds themselves (excluding their metabolites and by-products)not result directly in terminal toxicity. Therefore, membrane toxicity could be manifested as membrane blocking and membrane expansion. The method established here may be useful for evaluating the interaction of toxins with membranes. 


\section{Background}

Many toxic chemicals, e.g. drugs and harmful additives that are formed or utilized in daily life, accumulate persistently in biological systems. Sudan red compounds I IV, which are hydrophobic azo dyes, are still used as food additives in some countries because of their low cost and bright color [1] but they have been shown to be unsafe, causing tumors in the liver or urinary bladder [2] in rats. Sudan I - IV have been classified as category 3 carcinogens to humans by International Agency for Research on Cancer [3], and the European Union does not allow Sudan dyes as food additives [4,5]. A number of hypotheses that could explain the mechanism of carcinogenesis have been proposed for dyes similar to the Sudan red compounds [6].

Traditionally, investigations of lipid peroxidation damage by Sudan red compounds have been performed only on mouse and rabbit sera. Recently, Stiborova [7] compared experimental animals with humans and determined that cytochromes P450 and live microsomes are essential for extrapolating animal carcinogenicity data to human health risk assessment. Although Sudan dyes are indirect carcinogens, they generate metabolites that are converted to several active mutagens and carcinogens in humans $[8,9]$, such as aniline or 1-amido-2-naphthol, which can be metabolized by hepatic microsomes into benzene and naphthol. These end products can then combine with DNA and RNA to destroy cells [10].

To cause terminal toxicity, a chemical must penetrate the cell so as to affect the function of a target biomolecule. However, the cell membrane, which consists of a lipid bilayer and membrane proteins along with sugar polymers as a support layer, acts as a natural barrier and often plays a protective role in normal cellular activity. Cell membranes perform a number of essential functions such as transport of nutrients, ion conduction, signal transduction, cell migration, etc. The plasma membrane represents the primary permeability barrier of a cell and therefore regulates the inflow of substrates and the outflow of products. Normal cell activity will be seriously compromised if the cell is persistently exposed to a toxic medium. The partitioning of hydrocarbons in membrane buffer systems has been studied $[11,12]$. The available data show that because of their lipophilic character, these compounds interact with hydrophobic parts of the cell, and this is important in the mechanism of toxicity. Lipophilic compounds accumulate in the membrane, causing "expansion" of the membrane surface area, inhibition of primary ion pumps and increased proton permeability [12].

The interaction of lipophilic compounds with the phospholipid bilayer causes dramatic changes in cell membrane structure. For example, accumulation of such compounds in the hydrophobic part of the membrane will disturb the interactions between the phospholipid acyl chains, modifying membrane fluidity and eventually leading to swelling of the bilayer. Furthermore, the lipid annuli that surround membrane-embedded proteins will also change, possibly altering protein conformations [13]. Because Sudan red compounds have groups capable of forming hydrogen bonds, their toxic effects on the membrane should differ from those of apolar hydrocarbons.

In this study, we have investigated the interactions of the amphiphilic compounds Sudan II and IV with both lecithin liposomes and live E. coli membrane phospholipids. Polar compounds with hydrogen-bond forming groups e.g. - $\mathrm{OH},=\mathrm{O},-\mathrm{NH}_{2},-\mathrm{N}=\mathrm{N}$ - have been shown to accumulate on the external surfaces of membranes according to the Langmuir adsorption isotherm [14]. Entry into the liposome center and cytosol is impeded for amphiphilic compounds, so it is possible for them not to cause the terminal toxicity. However, their metabolites and by-products could be terminally toxic, possibly formed by exoenzyme or photocatalysis [15-17]. Membrane toxicity could therefore be manifested as membrane blocking and membrane expansion. The method established in the present work could be valuable for evaluating the interaction of toxins with membranes and will be applicable to research in the environmental and life sciences, and medicine.

\section{Results and discussion Absorption spectra and structural analysis}

Because Sudan II and IV are amphiphilic compounds, their absorption spectra were measured in ethanol solution (Figure 1) and showed wavelength maxima of 495 $\mathrm{nm}$ for Sudan II and $515 \mathrm{~nm}$ for Sudan IV. Both compounds contain the polar groups $-\mathrm{OH}$ and $-\mathrm{N}=\mathrm{N}-$-, which may form hydrogen bonds with the other polar groups. A methyl group of Sudan II is replaced by a methylphenylazo group in Sudan IV, extending the conjugation of double bonds, so that the $\lambda_{\max }$ of Sudan IV is increased. Since Sudan IV has one more lipophilic phenyl ring than Sudan II, Sudan IV is significantly less hydrophilic.

In order to investigate the interaction of these compounds with lecithin liposomes, the mixtures must be centrifuged. However, the centrifugal pellet appears light yellow in ethanol solution, so the background interference was estimated by a dual-wavelength correction [18] as follows:

$A_{\lambda 2}^{S / L}=\frac{a\left(A_{\lambda 2}-b A_{\lambda 1}\right)}{a-b}$

where 


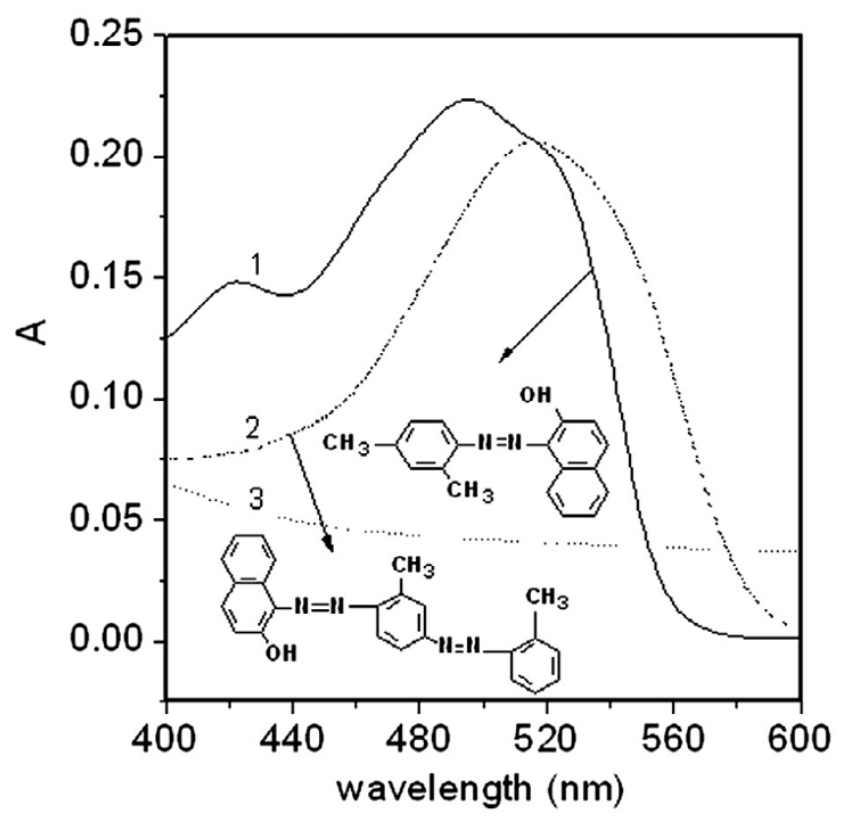

Figure I

Visible spectra of solutions containing: I, Sudan II (I4 $\mu \mathrm{M})$ in ethanol; 2, Sudan IV $(6.3 \mu \mathrm{M})$ in ethanol; 3, lecithin liposomes $(10 \mathrm{mg} / \mathrm{ml})$.

$a=\frac{A_{\lambda 2}^{S}}{A_{\lambda 1}^{S}}$ and $b=\frac{A_{\lambda 2}^{L}}{A_{\lambda 1}^{L}}$

and $\lambda_{1}$ and $\lambda_{2}$ are the measurement wavelengths. $\lambda_{1}$ was selected at $400 \mathrm{~nm}$ and $\lambda_{2}$ at $495 \mathrm{~nm}$ for Sudan II and 515 $\mathrm{nm}$ for Sudan IV. The symbol $A^{\mathrm{S} / \mathrm{L}}{ }_{\lambda 2}$ is the real absorbance of a Sudan compound adsorbed by the lecithin liposomes; $A_{\lambda 1}$ and $A_{\lambda 2}$ are the absorbances of the above solutions at $\lambda_{1}$ and $\lambda_{2}$. Both $a$ and $b$ are correction constants. $A^{L}{ }_{\lambda 1}$ and $A^{L}{ }_{\lambda 2}$ are the absorbances of a lecithin-ethanol solution at $\lambda_{1}$ and $\lambda_{2}$ without any Sudan compound and $A^{s}{ }_{\lambda 1}$ and $A^{S}{ }_{\lambda 2}$ are those of a Sudan compound in ethanol solution without liposomes. For Sudan II, $a=1.66$ and $b$ $=0.642 ;$ for Sudan IV, $a=2.73$ and $b=0.621$. These values were calculated from curves $1-3$ in Figure 1.

\section{Interaction of liposomes with the Sudan red compounds}

The absorptions of Sudan II and IV accumulated in liposomes are shown in Figure 2(A \& B). Their free concentrations in aqueous solution $\left(C_{\mathrm{L}}\right)$ were calculated from Eqs.2 and 4 in Table 1 . We attempted to analyze the interaction of liposomes with Sudan red compounds according to the Langmuir isothermal equation [14]:

$$
\frac{1}{\gamma}=\frac{1}{N}+\frac{1}{K N C_{L}}
$$

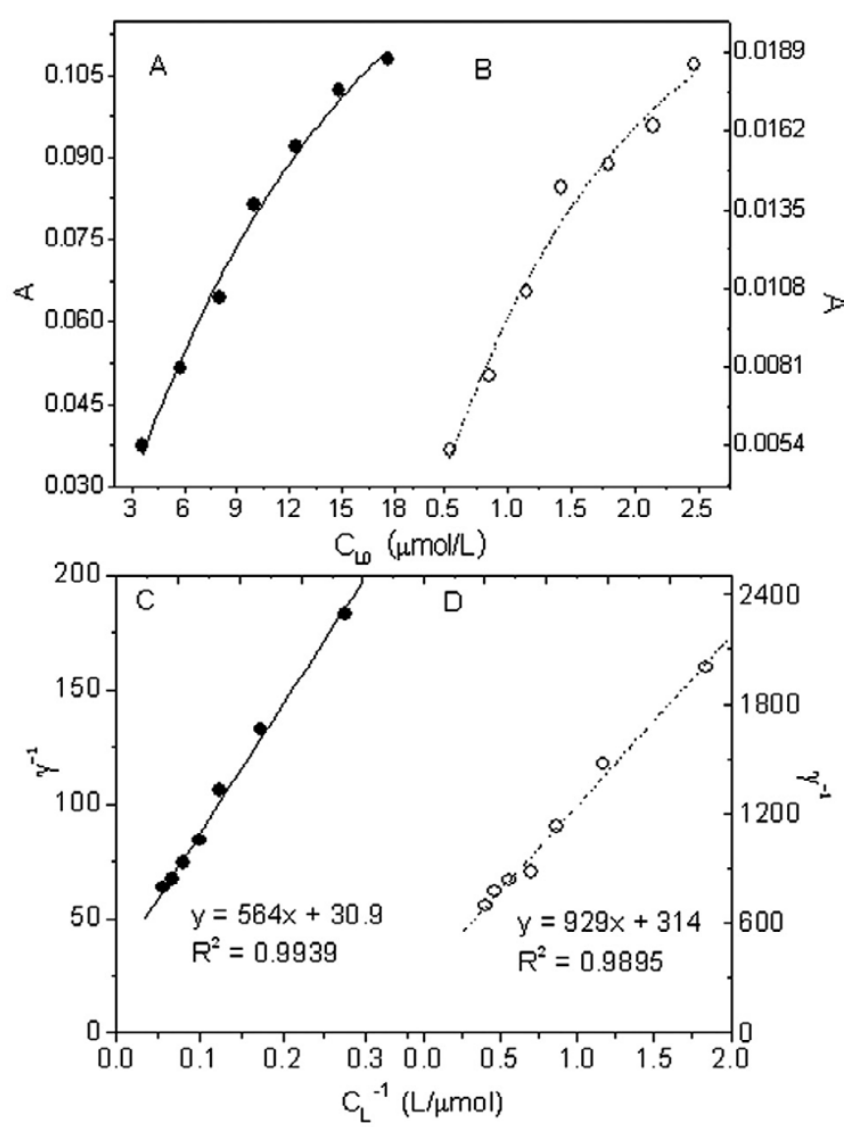

Figure 2

Absorbance measurements of the solutions containing $\mathbf{0 . 2 0} \mathbf{~ m g / m l}$ lecithin liposomes and Sudan II and IV and their linear regression. A: Sudan II in lecithin liposomes was dissolved in ethanol and the absorbance was measured at $495 \mathrm{~nm}$; B: Sudan IV was dissolved in ethanol and the absorbance was measured at $515 \mathrm{~nm}$; C: plots of $\gamma^{1}$ vs. $C_{L}{ }^{-1}$ for the interaction of Sudan II with liposomes; $D$ : as C, but using Sudan IV in place of Sudan II.

The symbol $\gamma$ denotes the binding number of $\mathrm{L}$ in liposomes and $N$ is the maximal binding constant. $K$ is the stability constant of the binding product. The higher the value of $K$ is and the stronger the binding becomes. Regression plots for $\gamma^{1}$ vs. $C_{\mathrm{L}}^{-1}$ in the Sudan red compound-lecithin liposome solutions (Figure 2(C and D)) indicated an interaction that obeyed the classical adsorption isotherm, with $N_{\text {Sudan-II }}=1 / 31$ and $K_{\text {Sudan-II }}=1.75 \times$ $0^{4}$ for the Sudan II-liposome system and $N_{\text {Sudan-IV }}=1 / 314$ and $K_{\text {Sudan-IV }}=2.92 \times 10^{5}$ for the Sudan IV-liposome system. Thus, 31 of lecithin molecules bound with one of Sudan II, but 314 of lecithin molecules with one of Sudan IV. This 10 -fold difference is attributed to the fact that the Sudan IV molecule is larger and has a greater steric effect than Sudan II. In addition, the Sudan IV-liposome complex is more stable than the Sudan II-liposome complex, 
Table I: Standard curves for determination of Sudan red compounds in ethanol and pH 7.0 phosphate buffer solution (PBS)

\begin{tabular}{lccccc}
\hline Chemical & Upper limit, $\mathrm{nM}$ & Wave- length, $\mathrm{nm}$ & Medium & Linear equations & Linear correlation coefficient \\
\hline Sudan II & 40 & 495 & ethanol & $A=0.0155 C_{\mathrm{L}}(2)$ & 0.9982 \\
Sudan II & 1.2 & 495 & PBS $*$ & $A=0.0121 C_{\mathrm{L}}-0.001(3)$ & 0.9993 \\
Sudan IV & 0.6 & 515 & ethanol & $A=0.0318 C_{\mathrm{L}}-0.0018(4)$ & 0.9990 \\
Sudan IV & 5 & 515 & PBS & $A=0.0196 C_{\mathrm{L}}-0.0007(5)$ & 0.9990 \\
\hline
\end{tabular}

as indicated by their $K$ values. The possible reason is the greater number of polar and lipophilic groups (potential binding sites) in Sudan IV can form more hydrogen bonds with the lecithin headgroups as illustrated in Figure 3(1) and more lipophilic bonds with aliphatic group of lecithin. Sudan II binding to a liposome is sketched in Figure 3(2). The hydrogen bonds appear around the surface of the liposome to form a polar binding sphere. In contrast, Sudan II will tend to move towards the central hydrophobic core because its aryl groups interact hydrophobically with the lecithin. Because of the polar attraction of hydrogen bonds, Sudan II is difficult to insert into the lecithin liposome center. Therefore, Sudan II could be adsorbed around the inner surface of the lecithin liposome as shown in Figure 3(2).

The phospholipid micelle will bind a polar hydrophobic organic compound via polar bonds, e.g. ion pairing, hydrogen bonds and dispersion forces, corresponding to the molecular monolayer adsorption model. The units of various non-covalent bonds determine the binding type, binding position and interaction strength of an organic compound [19]. With respect to biological activity, noncovalent interactions are common because they are nonspecific. A more complicated molecule with more active polar groups, e.g. Sudan IV, often forms a more stable complex. Thus, Sudan IV binding to lecithin liposomes cannot be reversed easily. The binding of Sudan II and IV by liposomes indicates thatentry into the liposome center may be impeded for them, as further argued on the basis of the following experiments.

\section{Effect of ionic strength, $\mathrm{pH}$ and temperature}

The effects of solution $\mathrm{pH}$, electrolyte content and temperature on the interactions of Sudan red compounds with lecithin liposomes are shown in Figure 4. Curves A- 1 and A-2 show that $\gamma$ increases slowly when the $\mathrm{pH}$ increases from 2.18 to 10.38 . If lecithin behaves similarly to an ionic surfactant [20], increasing $\mathrm{pH}$ favors the aggregation of organic substances. In addition, the hydroxyl groups of Sudan red compounds will form -O-anions in an alkaline media and will therefore bind firmly to the liposome surface by electrostatic interaction with the cationic lecithin headgroups. From curves B-1 and B-2, $\gamma$ decreases with increasing ionic strength. Again comparing lecithin with an ionic surfactant, increasing ionic strength is often unfavorable for the aggregation of soluble organic substances [20]. The aggregation number of lecithin in a liposome micelle increase seriously with an increase of electrolyte concentration [21]. The number of liposomes suspending in solution will decrease then the total spherical surface of micelles area decreases. One Sudan red molecule will cover more lecithin molecules though increasing ionic strength may be favorable for hydrophobic Sudan red

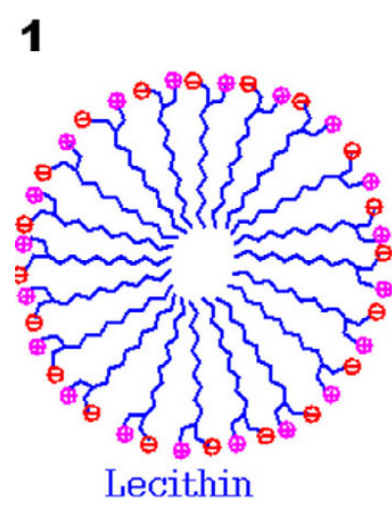

liposomes

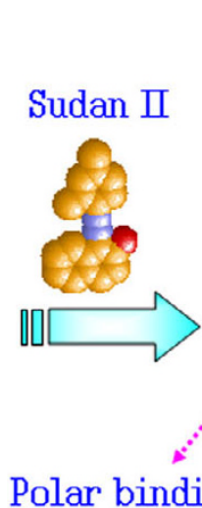

shell

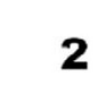

2 $2 \quad$ Hydrophobic 3

Figure 3

Cartoon illustrating the proposed Sudan red-liposome binding. I, Liposome sphere; 2, Sudan II - liposome binding pattern involving hydrogen bond and hydrophobic bond; 3, Sudan II - cell membrane binding pattern. 


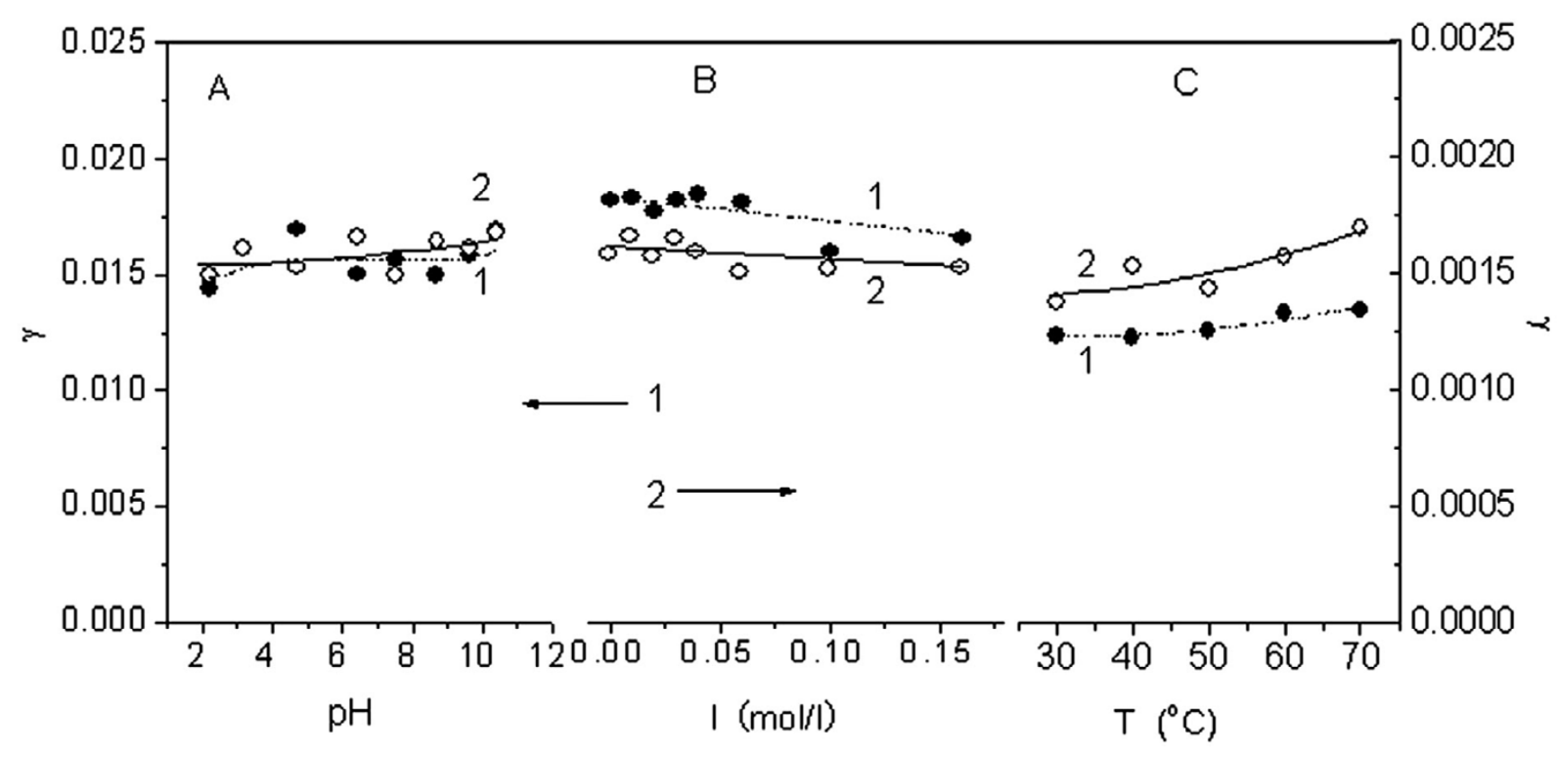

Figure 4

Effects of pH (A), electrolyte (B) and temperature (C) on the interactions of Sudan red compounds with lecithin liposomes (0.20 $\mathbf{m g} / \mathrm{I})$. I: $8 \mu \mathrm{M}$ Sudan II and 2: I $\mu$ M Sudan IV.

compounds to aggregate on the liposome. Curves C-1 and $\mathrm{C}-2$ show that $\gamma$ increases with increasing temperature. This is because the mean intermolecular distance in the liposome micelle increases at higher temperatures. Thus, the surface area of the lecithin liposome will increase, resulting in the binding of more Sudan red compounds.

\section{Analysis of E. coli growth state}

In order to select E. coli in stable growth, the number of viable cells was measured at $555 \mathrm{~nm}$ by methylthiazolyldiphenyltetrazolium (MTT) assay [22] as shown in Figure 5 . From curve 1 , the number of $E$. coli cells increases exponentially during the first $20 \mathrm{~h}$ and then remains constant between 20 and 36 h. After 36 h, cell death occurs. From curve 2, the absorption of the culture medium at $600 \mathrm{~nm}$ remains almost constant between 20 and $36 \mathrm{~h}$ and then increases after $36 \mathrm{~h}$. Curves 1 and 2 demonstrate not only that almost all the $E$. coli cells are alive but also that few cells were proliferating during the 20-36 h interval. Therefore, the cells were sampled at around $20 \mathrm{~h}$ for experiments to investigate the interactions between living cells and the Sudan red compounds.

\section{Distribution of Sudan red compounds in E. coli}

The toxicant in the extracellular medium enters the cells when possible by passive diffusion [23] and accumulates until the cell dies. In this work, the mixture of Sudan red compounds with E. coli was sampled at $30 \mathrm{~min}$ of culture time and treated according to standard procedures. Over
$98 \%$ of the E. coli in suspensions (1) and (4) was pelleted by centrifugation at $3500 \times \mathrm{g}$ for $5 \mathrm{~min}$, as determined by comparing the absorptions before and after centrifuga-

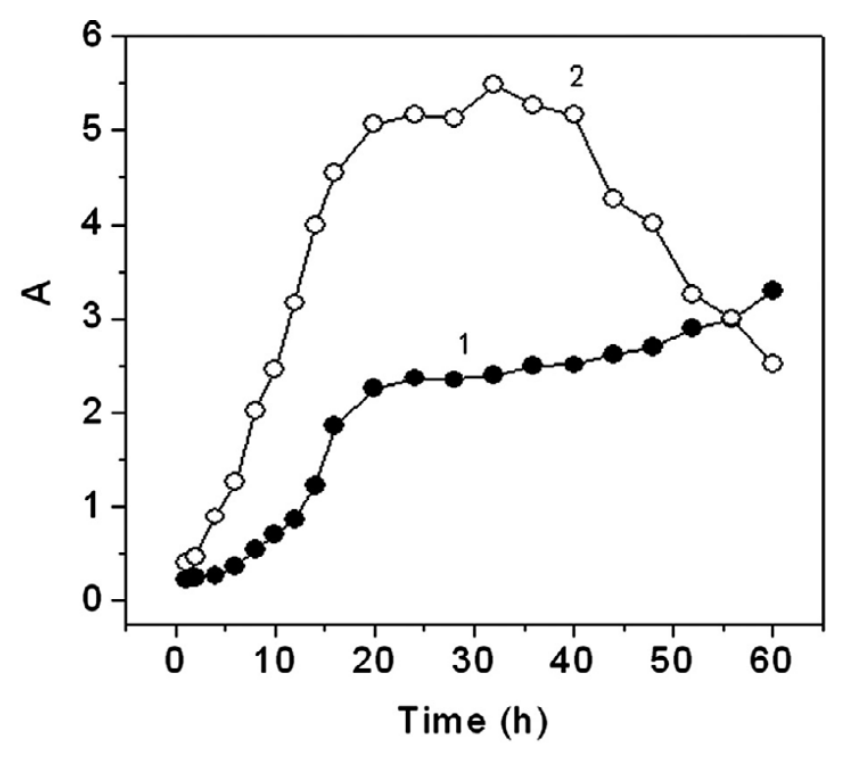

Figure 5

Growth curve of $\mathrm{E}$. coli at $37^{\circ} \mathrm{C}$. I: turbidity of culture fluid at $600 \mathrm{~nm}$ at $48 \mathrm{~h}$ culture time; 2 : variation of $E$. coli activity indicated by the absorbance measured at $555 \mathrm{~nm}$ with MTT. 
tion. Similarly, over $98 \%$ of the ultrasonication fragments in suspension (8) could be separated by centrifugation for $5 \mathrm{~min}$ at $5600 \times \mathrm{g}$. After fractionation, the absorptions of the Sudan red compounds in the extracellular solution, membrane and cytosol were measured as shown in Figure 6(A). Using Eqs. 2 - 5 in Table 1, the distributions of the Sudan red compounds were calculated as shown in Figure $6(\mathrm{~B} \& \mathrm{C})$. Over $60 \%$ of the Sudan II in the medium penetrated into the cell; $90 \%$ of this remained in the membrane. Similarly, from the columns in C, more than $75 \%$ of the Sudan IV entered the cells and about 95\% remained in the membrane. This results from the Langmuir adsorption of Sudan red compounds on to the membrane phospholipids via non-covalent interactions as described above. The binding model is sketched in Figure 3(3). As a
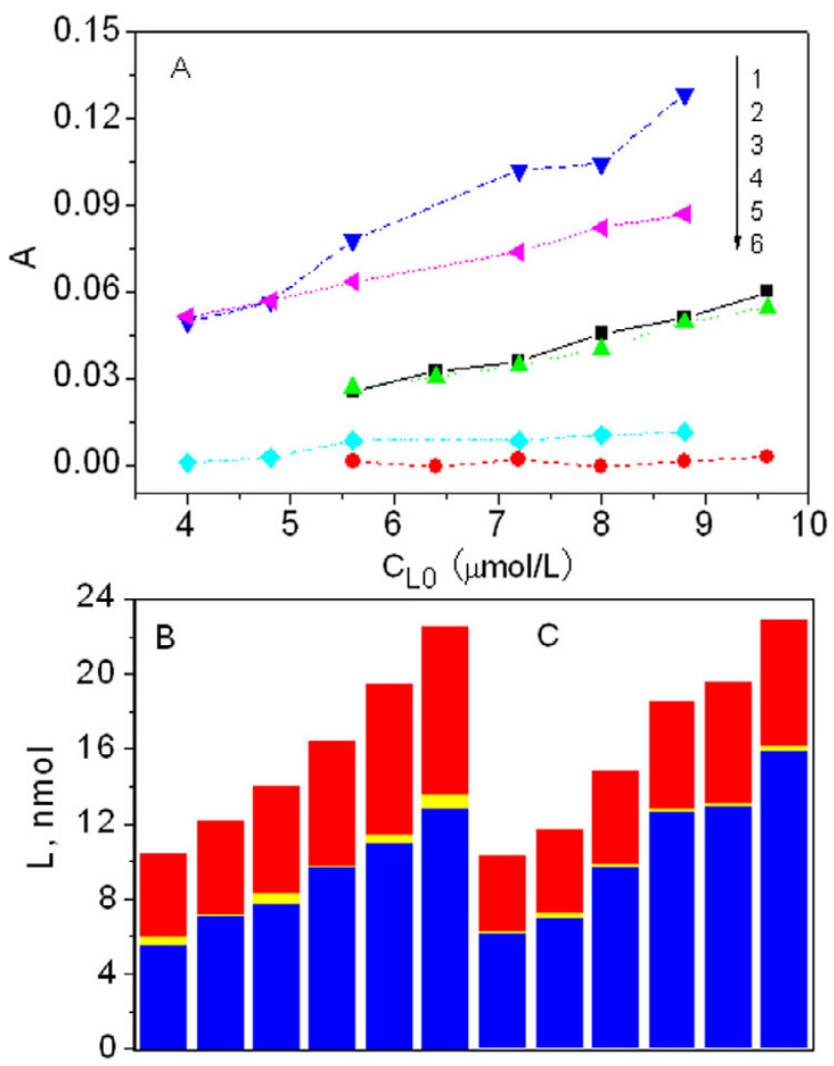

Figure 6

Spectrophotometric measurements of the solutions containing E. coli and Sudan red compounds. I: absorption of Sudan IV in extracellular solution; 2: absorption of Sudan IV accumulated in membrane; 3: absorption of Sudan II free in extracellular aqueous medium; 4: absorption of Sudan II accumulated in membrane; 5: absorption of Sudan IV in cytosol; 6: absorption of Sudan II in cytosol. B: distribution of Sudan II in the extracellular solution (red), membrane bilayer (blue) and cytosol (yellow). C: the equivalent information as given in B, but for Sudan IV. result, only a small amount of Sudan red compounds enters the E. coli cytosol, as shown by the small yellow parts of Figure 6(B and C), i.e. entry into the cytosol may be impeded for Sudan red compounds. While it is possible for such compounds to cause membrane toxicity, it is difficult to identify the target biomacromolecular toxicity, e.g. DNA damage, directly.

\section{Interaction of Sudan red compounds with cells}

In order to investigate the type of interaction between the Sudan red compounds and the membrane, the membrane phospholipids must be identified. The membrane was isolated according to standard procedures and the final preparation was dissolved in dichloromethane. The molar quantity of phosphorus in the membrane was determined by inductively coupled plasma - optical emission spectroscopy (ICP-OES) [24]. The results showed that $5 \mathrm{ml}$ of E. coli culture medium contained $1.32 \mu \mathrm{mol}$ of membrane phosphorus. From this, the values of $\gamma$ in the Sudan compound-E. coli mixtures were calculated, and plots of $\gamma^{1} \mathrm{vs}$. $C_{\mathrm{L}}^{-1}$ are shown in Figure 7. Obviously, the aggregation of Sudans II and IV in the cell membranes obeyed the Langmuir adsorption isotherm, as in the liposome prepara-

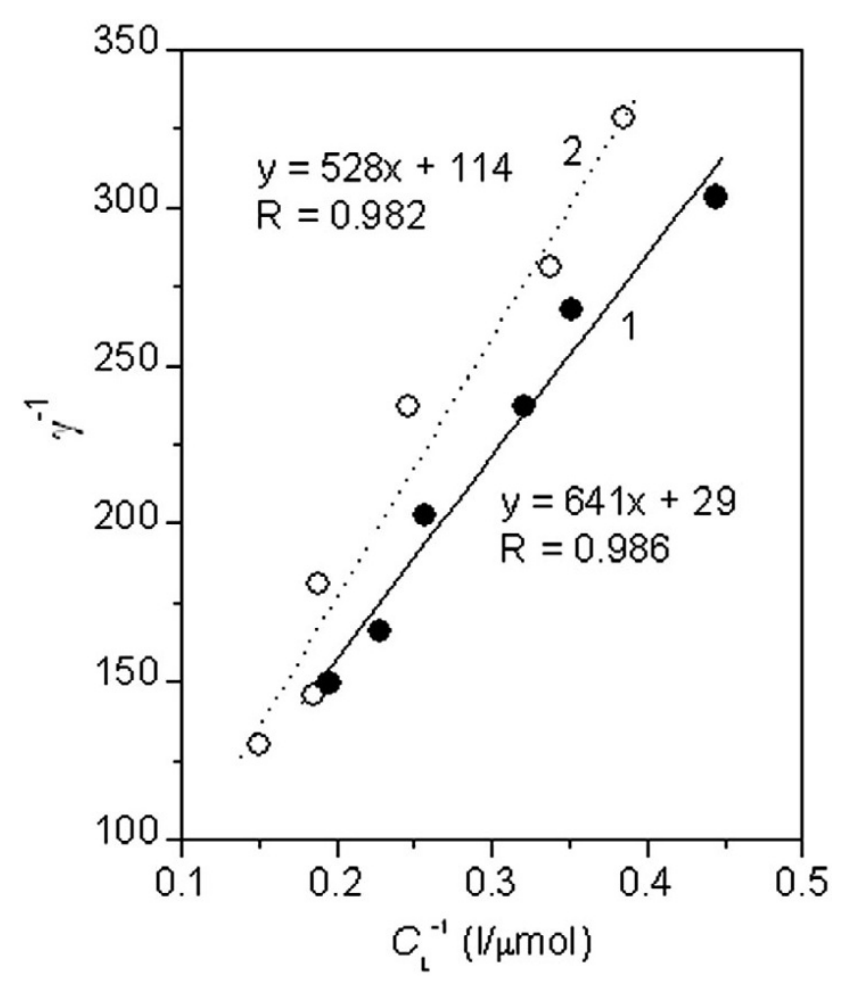

Figure 7

Linear regression curves for the interactions of Sudan red compounds with E. coli: A: Sudan II; B: Sudan IV. 
tions. The binding constants were calculated to be $N_{\text {Sudan }}$ II $=1 / 29, N_{\text {Sudan-IV }}=1 / 114$ and $K_{\text {Sudan-II }}=1.86 \times 10^{4}, K_{\text {Sudan- }}$ $\mathrm{IV}=6.02 \times 10^{4}$. The binding of Sudan IV in the cell membrane is more stable than that of Sudan II but a greater molar amount of Sudan II is bound. Comparison with Figure 2 shows that the $N$ and $K$ values of Sudan II in the cell membrane are very similar to those in the lecithin liposomes. For Sudan IV, however, $N$ is higher and $K$ is lower in the living cell membrane than in the lecithin liposomes. The membrane proteins may decrease the steric effect of the long Sudan IV molecules and increase the area covered. In addition, cell movement will continuously alter the surface-binding position and pattern of the long Sudan IV molecules in order to accommodate the adsorption of more of these molecules.

\section{Conclusion}

Penetration into the cytosol is impeded for Sudan red compounds, and it is possible for them not to cause the terminal toxicity. This work provides a useful experimental strategy for the quantitative assessment of potential membrane toxicity of hydrophobic compounds with polar groups and recognition of the terminally toxic substances. This technique may be useful in a variety of research fields including environmental and life sciences, and medicine.

\section{Methods \\ Materials and instruments}

Lecithin (Sinopharm Cheam), $20 \mathrm{mg} / \mathrm{ml}$, was suspended in deionized water and then dispersed ultrasonically at maximal amplitude at $4{ }^{\circ} \mathrm{C}$ for 5 cycles of $15 \mathrm{~s}$ interspersed with $45 \mathrm{~s}$ periods of rest [12]. Stock solutions of Sudan II (0.80 mM) and Sudan IV (0.10 mM) (Shanghai Chemical Reagents Center of National Medicine Group of China) were prepared in absolute ethanol. Britton-Robinson (BR) buffers at pH 2.18, 3.13, 4.16, 5.72, 7.16, 8.69, 9.62 and 10.38 were prepared and were used to examine the effect of solution $\mathrm{pH}$ on the interactions of the Sudan red compounds with liposomes. A pH 7.0 PBS was prepared by mixing $0.8 \% \mathrm{NaCl}, 0.02 \% \mathrm{KCl}, 0.0144 \% \mathrm{Na}_{2} \mathrm{HPO}_{4}$ and $0.024 \% \mathrm{KH}_{2} \mathrm{PO}_{4}$ and was used to re-suspend bacterial pellets. Sterile L-B nutrient medium containing 1.0\% peptone, $0.5 \%$ yeast extract and $1.0 \% \mathrm{NaCl}$ was prepared and used for culturing E. coli. The E. coli strain used was provided by the Environmental Biological Laboratory of Tongji University.

A Model Lambda-25 spectrometer (Perkin-Elmer Instruments, USA) with version-2.85.04 UV-WinLab software was used to record the absorption spectra of the Sudan solutions and to measure turbidities and absorptions. A Model JY92-II Ultrasonic Cell Disruptor (Ningbo Xinzhi Instruments, China) was used to disrupt the bacterial cells. A Model Anke TGL-16C High-speed Centrifuge
(Shanghai Anting Sci. Instruments, China) with $7.0 \mathrm{ml}$ plastic tubes was used to separate the liposomes and cell membranes. Solution pHs were measured with a Model pHS-25 Acidity Meter (Shanghai Precise Instruments., China). A Model TS-030 Thermostat Cycling Bath (Shanghai Yiheng S\&T, China) was used to maintain the experimental temperature between room temperature and $70^{\circ} \mathrm{C}$. A Model SK3300H Ultrasonic Cleaning Device (Shanghai Ultrasonic Cleaning Instruments, China) was used to accelerate the dissolution of solutes and to disperse the phospholipids. A Model CHA-2 Thermostat Gas Bath Vibrator (Jintan E-tong Electrons, Jiangsu, China) and a Model SPX-80BS-II Biochemical Incubator (Shanghai Cimo Medical instruments, China) were used to culture the bacteria. A Model Optima 2100 DV ICP- OES (PerkinElmer Instruments, USA) was used to measure the phosphorus content of the membrane phospholipids. A Model GM-100 diaphragm vacuum pump (Tengda Filtration Devices of Tianjin, China) was used to volatilize the organic reagent in the membrane extracts to yield the $E$. coli membrane residue.

\section{In-vitro interaction of Sudan red compounds with liposomes}

A series of mixtures containing $50 \mu \mathrm{l}$ of liposome suspension and aliquots of $0.8 \mathrm{mM}$ Sudan II ( 0 to $120 \mu \mathrm{l}$ ) were diluted to $5.00 \mathrm{ml}$ with deionized water. After mixing for $10 \mathrm{~s}$ in the ultrasonic cleaning device, they were centrifuged for $20 \mathrm{~min}$ at $7000 \times \mathrm{g}$. Each pellet was dissolved in $5.00 \mathrm{ml}$ of ethanol. A dual-wavelength spectral correction method [18] was used to eliminate background interference. The solutions were measured at 400 and $495 \mathrm{~nm}$, and a standard curve of Sudan II in the liposomes was generated. Applying the same procedures, a standard curve prepared from aliquots of the $0.1 \mathrm{mM}$ stock solution of Sudan IV was used to measure the interaction of Sudan IV with the liposomes, except that data were collected at 400 and $515 \mathrm{~nm}$.

\section{Culture of E. coli and determination of membrane phospholipids}

E. coli was selected as the model microorganism for further experiments with the Sudan dyes. Several cycles of the E. coli strain were grown in $250 \mathrm{ml}$ of L-B nutrient medium in culture bottles in the thermostated gas bath vibrator at $37^{\circ} \mathrm{C}$. Bacterial growth was monitored by turbidimetric measurement at $600 \mathrm{~nm}$. The E. coli cultures were also treated with MTT [22] and the absorbance measured at $555 \mathrm{~nm}$ to determine the number of living cells.

After the E. coli growth reached stationary phase, $5.00 \mathrm{ml}$ of the zymotic fluid from the culture bottle was placed in a 7-ml centrifuge tube. The sample was centrifuged for 5 min in $3500 \times \mathrm{g}$ to sediment the bacteria, and the yellow supernatant was removed. The pellet was resuspended 


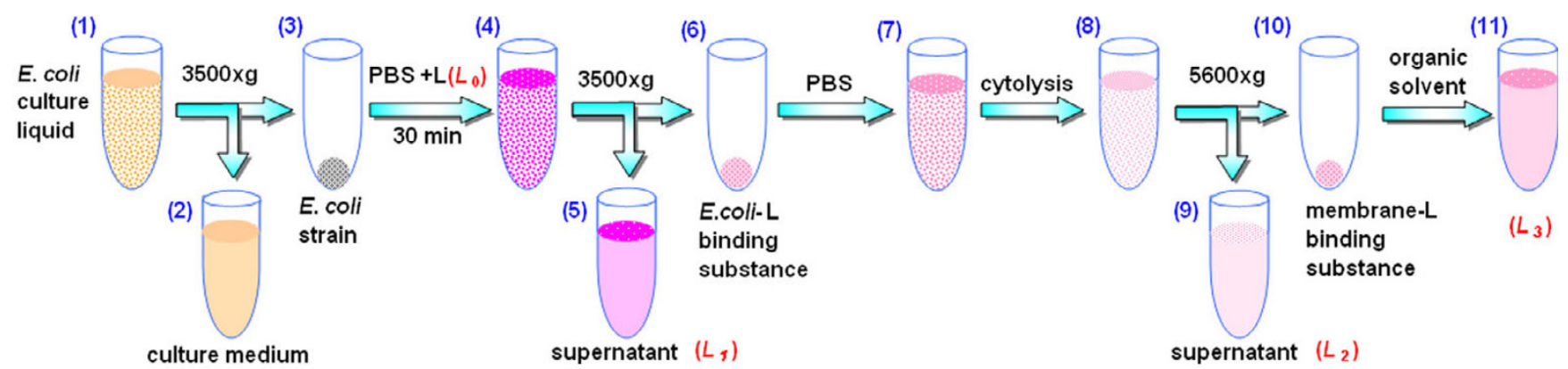

Figure 8

Cartoon illustrating the separation and detection procedures used to investigate the interaction between $E$. coli and Sudan red compounds (L). (I) E. coli culture medium; (2) supernatant of (I) after centrifugation at $3500 \times$ g; (3) $E$. coli collected; (4) E. coli liquid re-suspended with PBS still containing Sudan dyes (L) ; (5) supernatant of (4) after centrifugation at $3500 \times \mathrm{g}$; (6) E. coli - L binding substance collected; (7) $E$. coli - L binding liquid re-suspended with PBS; (8) $E$. coli $-L$ binding liquid after ultrasonication; (9) supernatant of (8) after centrifugation at $5600 \times \mathrm{g}$; (10) membrane $-\mathrm{L}$ binding substance collected and (I I) membrane $-\mathrm{L}$ binding solution dissolved in organic solvent.

with PBS (2.5 ml), mixed well, and centrifuged again to obtain a pellet. The pellet was suspended and dispersed in PBS $(2.5 \mathrm{ml})$ by ultrasonication for $10 \times 5 \mathrm{~s}$ at $200 \mathrm{w}$ interspersed by $10 \mathrm{~s}$ intervals of rest. After centrifugation, the supernatant was removed, the pellet was dissolved in 2.50 ml dichloromethane and the organic phase was volatilized under vacuum. The dried residue was treated [25] with $4 \mathrm{ml} \mathrm{H}_{2} \mathrm{SO}_{4}, 4 \mathrm{ml} \mathrm{HCl}$ and $5 \mathrm{ml}$ water to prepare a clear solution. The concentration of phosphorus was determined by ICP-OES [24]. From this, the molarity of the membrane phospholipid species in the E. coli culture medium was calculated.

\section{Interactions of Sudan red compounds with E. coli}

Five $\mathrm{ml}$ of the zymotic fluid was centrifuged for $5 \mathrm{~min}$ at $3500 \times \mathrm{g}$ (Figure 8) after E. coli growth reached stationary phase. The yellow supernatant was removed and the E. coli pellet (3) was re-suspended in $2.5 \mathrm{ml}$ of PBS. Aliquots (0$\left.120 \mu \mathrm{l}\left(L_{0}\right)\right)$ of Sudan II (L) were added. After incubation in an isothermal shaker bed at $37^{\circ} \mathrm{C}$ for $30 \mathrm{~min}$, the liquid was centrifuged for $5 \mathrm{~min}$ at $5600 \times \mathrm{g}$. The absorbance of the supernatant (5) was measured at $495 \mathrm{~nm}$. The amount $\left(L_{1}\right)$ of free Sudan II was calculated by Eq. 2 as shown in Table 1. The pellet (6) was again re-suspended in $2.5 \mathrm{ml}$ of PBS (7) and centrifuged as above, and the clear solution was removed. PBS $(2.5 \mathrm{ml})$ was added to the centrifuge tube and the cells were ruptured by ultrasonication for 5 $\times 5 \mathrm{~s}$ at $200 \mathrm{w}$ interspersed with $10 \mathrm{~s}$ rest periods. The liquid (8) was centrifuged for $5 \mathrm{~min}$ at $5600 \times \mathrm{g}$. The absorbance of the supernatant (9) was measured according to the above method and the amount $\left(L_{2}\right)$ of Sudan II in the cytosol calculated using Eq. 3. The pellet (10) was dissolved in $2.50 \mathrm{ml}$ of ethanol to prepare a clear solution. The amount $\left(L_{3}\right)$ of Sudan II accumulated in the membrane was calculated by Eq. 2 from the absorbance of this solution (11). Simultaneously, Sudan IV and a blank without $\mathrm{L}$ were processed by the same procedures, except that Eqs. 5 and 4 were used instead of Eqs. 3 and 2 to calculate the amounts of Sudan IV in the intracellular liquid, cytosol and membrane. Thus, the distributions of Sudan II and IV were determined.

\section{Authors' contributions}

LL performed all of the experimental operations and treated the data. HWG designed the experimental procedures, created the cartoon illustration and performed writing of the manuscript. JRR performed the preparation of lecithin liposome liquid and optimization of the centrifugation condition. LC collected and analyzed the data. YCL provided the financial support for this work. JFZ assisted the English editing and polishing of this manuscript. HPZ performed the culture of E. coli strain. YY provided the research tools for this work. All authors read and approved the final manuscript.

\section{Acknowledgements}

The authors sincerely thank the National Natural Science Foundation of China (No. 20477030) and the Shanghai Fundamental Research Projects (No. 04J Cl 4072 and 05JCl 4059) for financially supporting this work.

\section{References}

I. Ma M, Luo X, Chen B, Su S, Yao S: Simultaneous determination of water-soluble and fat-soluble synthetic colorants in foodstuff by high-performance liquid chromatography-diode array detection electrospray mass spectrometry. I Chromatogr A 2006, I 1 03: 170-176.

2. Golka K, Kopps S, Myslak ZW: Carcinogenicity of azo colorants: influence of solubility and bioavailability. Toxicol Lett 2004, 15 1:203-210.

3. Tateo F, Bononi M: Fast determination of Sudan I by HPLC/ APCI-MS in hot chilli, spices, and oven-baked foods. J Agr Food Chem 2004, 52:655-658.

4. Puoci F, Garreffa C, lemma F, Muzzalupo R, Spizzirri UG, Picci N: Molecularly imprinted solid phase extraction for detection of Sudan I in food matrices. Food Chem 2005, 93:349-353.

5. Donna LD, Maiuolo L, Mazzotti F, Luca DD, Sindona G: Assay of Sudan I Contamination of Food stuff by Atmospheric Pres- 
sure Chemical Ionization Tandem Mass Spectrometry and Isotope Dilution. Anal Chem 2004, 76:5 I04-5 I08.

6. Klaus G, Silke K, Zdislaw W: Carcinogenicity of azo colorants: influence of solubility and bioavailability. Toxicol Lett 2004, I5 I:203-210.

7. Stiborova M, Martinek V, Rvdlova H, Hodek P, Frei E: Sudan I Is a Potential Carcinogen for Humans: Evidence for Its Metabolic Activation and Detoxication by Human Recombinant Cytochrome P450 IAI and Liver Microsomes. Cancer Res 2002, 62:5678-5684.

8. Stiborova M, Asfaw B, Anzenbacher P, Leseticky L, Hodek P: The first identification of the benzenediazonium ion formation from a non-aminoazo dye, I-phenylazo-2-hydroxynaphthalene (Sudan I) by microsomes of rat livers. Cancer Lett 1988, 40:319-326

9. Childs JJ, Clayson DS: The metabolism of I-phenylazo-2-naphthol in the rabbit. Biochem Pharmacol 1966, 15:1247-1258.

10. Nesn S, Argus M, Bergman H, Chu K, Frith C, Helmes T, Mcgaughy R, Ray V, Slaga T, Tennant R, Weisburger E: Chemical carcinogenesis: a review and analysis of the literature of selected chemicals and the establishment of the gene-tox carcinogen data base: a report of the US. Environmental Protection Agency gen-tox program. Mutat Res 1987, I85: I- 195.

II. Sikkema J, Bont J, Poolman B: Interactions of cyclic hydrocarbons with biological membranes. J Biol Chem 1994, 269:8022-8028.

12. Saar K, Lindgren M, Hansen M, Eiríksdóttir E, Jiang Y, Rosenthal-Aizman K, Sassian M, Langel ülo : Cell-penetrating peptides: A comparative membrane toxicity study. Anal Biochem 2005, 345:55-65.

13. Sikkema J, Bont J, Poolman B: Mechanisms of membrane toxicity of hydrocarbons. Microbiol Rev 1995, 59:201-222.

14. Langmuir I: The adsorption of gases on plane surfaces of glass, mica and platinum. J Am Chem Soc 1918, 40: I36I-I402.

15. Kamer M, Rassoulzadegan F: Extracellular enzyme-activity indications for high short-term variability in a coastal marine ecosystem. Microb Ecol 1995, 30:143-156.

16. Wang F, Zheng TL, Hong HS: Ecological function of bacterial extracellular enzyme. Chin J Mar Sci 1999, 3:15-17.

17. Hong JL, Emori H, Otaki M: Photodecolorization of azo dyes by extracellular metabolites under fluorescent light and influence of operational parameters. J Biosci Bioengineer 2005 100:192-196.

18. Gao HW, Zhang PF: Determination of antimony in wastewater with chromazurol $\mathbf{S}$ by beta-correction spectrophotometry. Analyst 1994, I19:2109-2111.

19. Gao HW, Zhao JF, Yang QZ, Liu XH, Chen L, Pan LT: Non-covalent Interaction of 2', 4', 5', 7'-Tetrabromo-4, 5, 6, 7-Tetrachlorofluorescein with Proteins and Its Application. Proteomics 2006, 6:5|40-5|5|.

20. Gao HW, Xu WQ: Langmuir Aggregation of Evans Blue on Cetyltrimethylammonium Bromide and on Proteins and Application. Anal Chim Acta 2002, 458:417-424.

21. Maron SH, Lando JB: Fundamentals of physical Chemistry. Collier Macmillan Pub 1974:466-797.

22. Wei HG, Li YG, Liu J, Sheng GM, Wu JM: Studies on a new method for counting living bacterial cell number. Chin J Microbiol 2002, 29:89-93.

23. Kansy M, Senner F, Gubernator K: Physicochemical high throughput screening: parallel artificial membrane permeation assay in the description of passive absorption processes. J Med Chem 1998, 41:1007-1010.

24. Ruiz V, Ribeiro AS, Airoldi C: A new elemental analysis procedure based on an ICP-OES technique to determine arsenic, phosphorus and titanium in titanium phenylphosphonate or titanium phenylarsonate. Curr Anal Chem 2005, I: I7I-I75.

25. Zhou M, Struve DM: The effects of post-persulfate-digestion procedures on total phosphorus analysis in water. Water Res 2004, 38:3893-3898.
Publish with Bio Med Central and every scientist can read your work free of charge

"BioMed Central will be the most significant development for disseminating the results of biomedical research in our lifetime. "

Sir Paul Nurse, Cancer Research UK

Your research papers will be:

- available free of charge to the entire biomedical community

- peer reviewed and published immediately upon acceptance

- cited in PubMed and archived on PubMed Central

- yours - you keep the copyright
BioMedcentral 